\title{
Bilayer of Terbium Double-Decker Single-Molecule Magnets
}

\author{
Giulia Serrano, ${ }^{* \dagger}$ Stefan Wiespointner-Baumgarthuber, ${ }^{\dagger}$ Stefano Tebi, ${ }^{\dagger}$ Svetlana Klyatskaya, \\ Mario Ruben, ${ }^{\ddagger}, \S$ Reinhold Koch, ${ }^{\dagger}$ and Stefan Müllegger ${ }^{\dagger}$ \\ ${ }^{\dagger}$ Institute of Semiconductor and Solid State Physics, Johannes Kepler University, 4040 Linz, Austria \\ ${ }^{\ddagger}$ Institute of Nanotechnology, Karlsruhe Institute of Technology, 76344 Eggenstein-Leopoldshafen, Germany \\ ${ }^{\S}$ Institut de Physique et de Chimie des Materiaux de Strasbourg, Universite de Strasbourg, CNRS UMP 7504, 23 Rue du Loess, \\ Strasbourg 67034 Cedex 2, France
}

Supporting Information

ABSTRACT: We report a low-temperature scanning tunneling microscopy and spectroscopy study of the structural and electronic properties of a bilayer of terbium double-decker (bis(phthalocyaninato)terbium(III), $\mathrm{TbPc}_{2}$ ) molecules on $\mathrm{Au}(111)$ at $5 \mathrm{~K}$. The $\mathrm{TbPc}_{2}$ molecules are found to adsorb flat on top of a first compact $\mathrm{TbPc}_{2}$ monolayer on $\mathrm{Au}(111)$, forming a square-like packing similar to the underlying first layer. Their frontier-orbital electronic structure, measured by tunneling conductance spectroscopy, clearly differs from that of the underlying first monolayer. Our results of second-layer molecules indicate the absence of, both, hybrid moleculesubstrate electronic states close to the Fermi level and a zerobias Kondo resonance. We attribute these findings to a decreased electronic coupling with the $\mathrm{Au}(111)$ substrate.

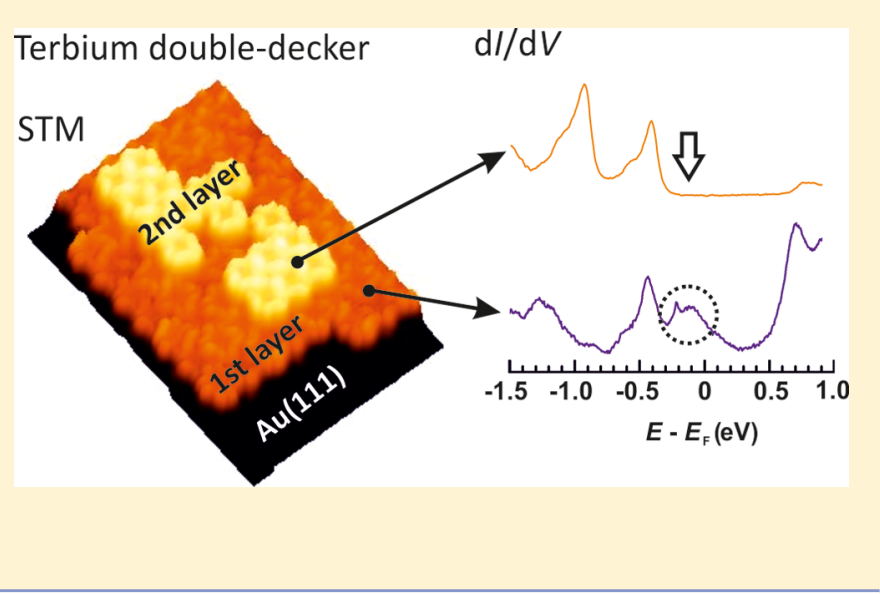

which have been found to lower the electronic interaction between molecules and metal substrates. Moreover, decreased electronic coupling of molecules and substrates has been reported for multilayer coverages of (metal-) organic functional molecules such as porphyrins, ${ }^{16}$ phthalocyanines, ${ }^{17}$ and $\mathrm{C}_{60}{ }^{18}$ on different surfaces.

Here, we investigate an alternative strategy for lowering the coupling of $\mathrm{TbPc}_{2}$ molecules to a metal template, utilizing a compact ordered monolayer of $\mathrm{TbPc}_{2}$, as a spacer layer, on top of which additional $\mathrm{TbPc}_{2}$ molecules are adsorbed. Their topographical and electronic properties are investigated by scanning tunneling microscopy (STM) and - spectroscopy. While the structural and electronic properties of $\mathrm{TbPc}_{2}$ have been studied by STM on different substrates including $\mathrm{Au}(111),{ }^{2,19-21} \mathrm{Cu}(111),{ }^{6} \mathrm{Co}(111),{ }^{22} \mathrm{Ir}(111),{ }^{23}$ highly ordered pyrolytic graphite, ${ }^{10}$ and $\mathrm{H}-\mathrm{Si}(001),{ }^{5}$ reports on bilayer $\mathrm{TbPc}_{2}$ are still scarce. Our results indicate that the $\mathrm{TbPc}_{2}$ molecules of the second layer lie in a flat manner on the spacer layer and form a square-like packing similar to the underlying monolayer but are weakly coupled to the $\mathrm{Au}(111)$ substrate.

Received: April 11, 2016

Revised: $\quad$ May 17, 2016

Published: May 18, 2016 


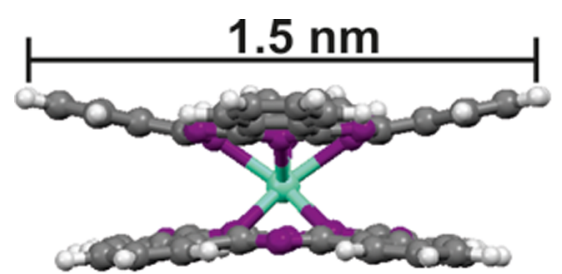

Side view

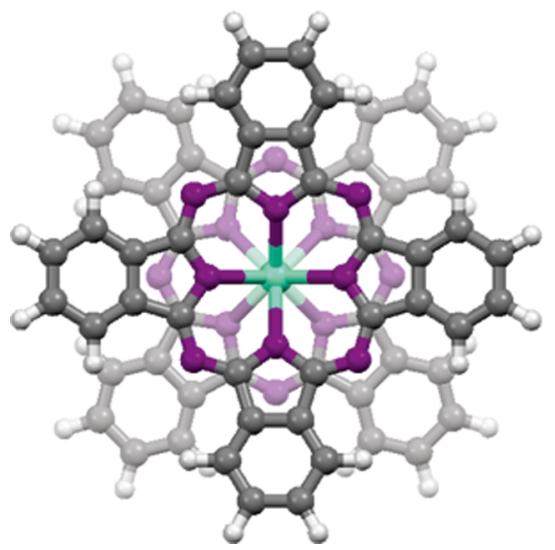

Top view

Hydrogen

Carbon

Nitrogen

Terbium

Figure 1. Schematic model of a terbium double-decker molecule $\left(\mathrm{TbPc}_{2}\right)$. The bottom phthalocyanine ligand is displayed in transparency for clarity (top view).

\section{METHODS}

The single-crystal $\mathrm{Au}(111)$ substrate, exhibiting the well-known $22 \times \sqrt{3}$ herringbone reconstruction, was prepared by repeated cycles of $0.6 \mathrm{keV} \mathrm{Ar}^{+}$ion bombardment and annealing at about $700 \mathrm{~K}$. A neutral form of $\mathrm{TbPc}_{2}$ was obtained by modified protocol based on templating reactions starting from a mixture of the phthalonitrile precursor o-dicyanobenzene and the terbium acetylacetinate $\mathrm{Tb}(\mathrm{acac}) \cdot n \mathrm{H}_{2} \mathrm{O}$, in the presence of a strong base (e.g., DBU) and high-boiling solvents, such as hexanol. ${ }^{24}$ Analytically pure powder samples were obtained with $30 \%$ yield. Deep green crystals of the products can be produced by using slow diffusion of $\mathrm{CH}_{2} \mathrm{Cl}_{2}$ into $\mathrm{C}_{2} \mathrm{H}_{2} \mathrm{Cl}_{4}$ solution of the pristine material. The $\mathrm{TbPc}_{2}$ crystallized in the space group (-phase) in the same way as reported by Takeya et al. ${ }^{20} \mathrm{TbPc}_{2}$ was thermally evaporated from a quartz crucible at a source temperature of $700 \mathrm{~K}$ and a pressure of less than $1 \times$ $10^{-9}$ mbar onto the $\mathrm{Au}(111)$ substrate held at room temperature, after thorough degassing the molecules for $>12 \mathrm{~h}$ at $363 \mathrm{~K}$ and $<1 \mathrm{~h}$ at $473 \mathrm{~K}$. STM experiments were performed at $5 \mathrm{~K}$ and a base pressure below $5 \times 10^{-11} \mathrm{mbar}$ employing electrochemically etched $\mathrm{W}$ tips deoxidized by annealing above $1100 \mathrm{~K}$ in vacuum. The bias voltage was applied to the sample. The differential tunneling conductance, $d I / d V$, signal was obtained from the first harmonic current signal detected by lock-in technique with sinusoidal modulation of the sample bias at $704 \mathrm{~Hz}$ and $10 \mathrm{mV}$ zero-to-peak. $d I / d V$ spectra were recorded at constant-height conditions after deactivating the STM feedback at a set point of $+1 \mathrm{~V}$ and $50 \mathrm{pA}$ with $z$-offset $\leq 85 \mathrm{pm}$ and sweep time of $40 \mathrm{~s}$. Impurity and tip effects were minimized by multiple tip forming (controlled indentation) on the pristine Au substrate between the experiments, resulting in Au-coated tips. Reliable tip performance was established by accurately reproducing the characteristic conductance signature of the $\mathrm{Au}(111)$ surface state well-known in the literature.

\section{RESULTS AND DISCUSSION}

The $\mathrm{TbPc}_{2}$ molecule is a double-decker complex, where a single $\mathrm{Tb}^{3+}$ ion is sandwiched by two phthalocyanine ligands; the two ligands are oppositely domed (concave and convex) and rotated by $45^{\circ}$ with respect to each other (Figure 1$) .{ }^{20}$ On a $\mathrm{Au}(111)$ surface, $\mathrm{TbPc}_{2}$ adsorbs flat, i.e., with the ligand planes parallel to the substrate surface-as previously reported for individual molecules as well as ordered molecular film. ${ }^{20}$ Figure $2 \mathrm{a}$ shows an STM image obtained after depositing $\mathrm{TbPc}_{2}$

(a)

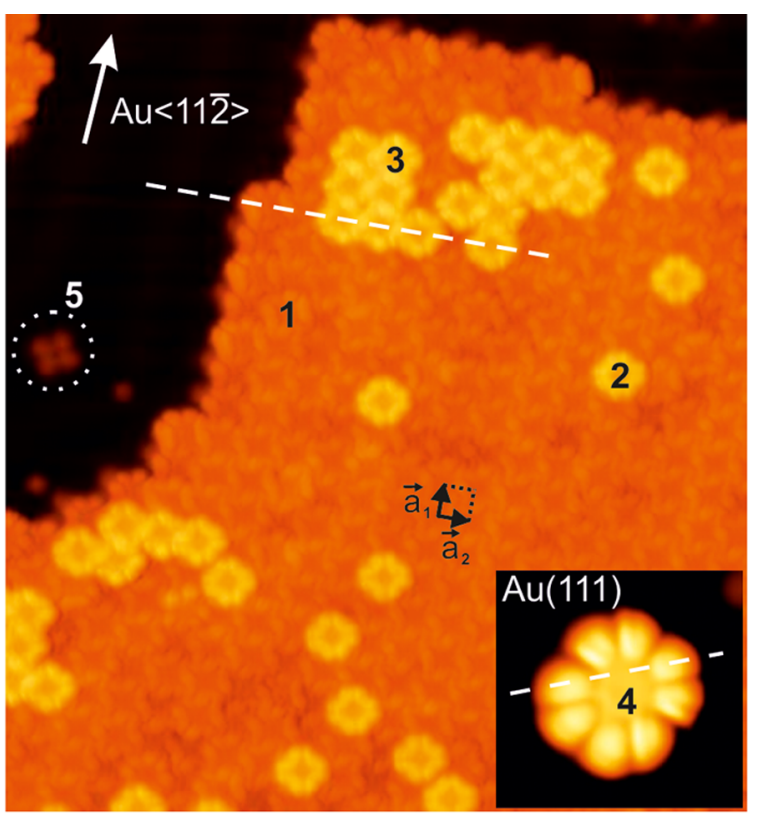

(b)

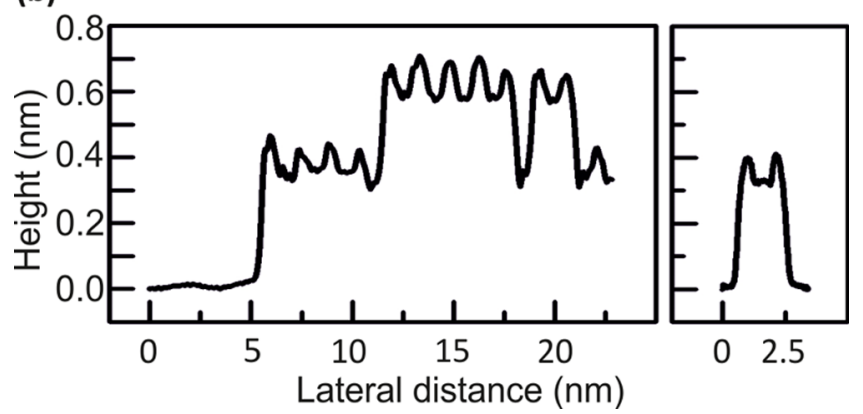

Figure 2. (a) STM image of $\mathrm{TbPc}_{2}$ molecules on $\mathrm{Au}(111)$ at a coverage of approximately $80 \%\left(25 \times 27 \mathrm{~nm}^{2}, 40 \mathrm{pA},+0.9 \mathrm{~V}\right)$; arrow marks $\mathrm{Au}\langle 11 \overline{2}\rangle$ direction; inset: STM image of a single isolated $\mathrm{TbPc}_{2}$ molecule on $\mathrm{Au}(111), 3 \times 3 \mathrm{~nm}^{2}, 50 \mathrm{pA},+0.9 \mathrm{~V}$; labels: see text. (b) Topographic STM height profiles; left: profile across the film of 1stand 2nd-layer $\mathrm{TbPc}_{2}$ molecules along the dashed line in (a); right: profile across a single $\mathrm{TbPc}_{2}$ molecule on $\mathrm{Au}(111)$ along the dashed line in the inset of (a).

molecules on $\mathrm{Au}(111)$ with approximately $80 \%$ of coverage. The dark area of the image corresponds to the bare substrate; the $\mathrm{Au}\langle 11 \overline{2}\rangle$ (herringbone) direction is indicated by an arrow. The large bright (orange) region, labeled 1, represents an ordered film of $\mathrm{TbPc}_{2}$ molecules lying directly on $\mathrm{Au}(111)$ and denoted herein as first-layer film. Molecules of the first-layer film form a square-like packing (about $1.4 \times 1.4 \mathrm{~nm}^{2}$ ) as reported earlier; ${ }^{20,24}$ respective lattice vectors are labeled $\vec{a}_{1}$ and $\vec{a}_{2}$. On top of the first-layer film, additional (bright) protrusions are imaged by STM (Figure 2a), exhibiting size and shape very 
similar to that of first-layer $\mathrm{TbPc}_{2}$ molecules. Apparently, additional $\mathrm{TbPc}_{2}$ molecules lie on top of the first-layer film, either as individual molecules (labeled 2) or as part of a molecular film (labeled 3).

In order to unambiguously corroborate this attribution of type 2 and type 3 molecules, we have carefully analyzed our topographical STM images. Figure 2b, left, shows a representative topographical profile across the film along the dashed line in Figure 2a. The profile shows that molecules in the first-layer film (type 1) have an almost identical height of $0.4 \mathrm{~nm}$ compared to individual single $\mathrm{TbPc}_{2}$ molecules on $\mathrm{Au}(111)$. The latter is shown in the inset of Figure 2a (type 4); the respective profile of a single $\mathrm{TbPc}_{2}$ molecule (dashed line in the inset of Figure 2a) is shown in Figure 2b, right. In contrast, type 2 and type 3 molecules lying on top of the first-layer film exhibit a height of about $0.7 \mathrm{~nm}$ with respect to the $\mathrm{Au}(111)$ substrate. This value is slightly lower than the expected value of $0.8 \mathrm{~nm}$, corresponding to two times the first-layer height. We
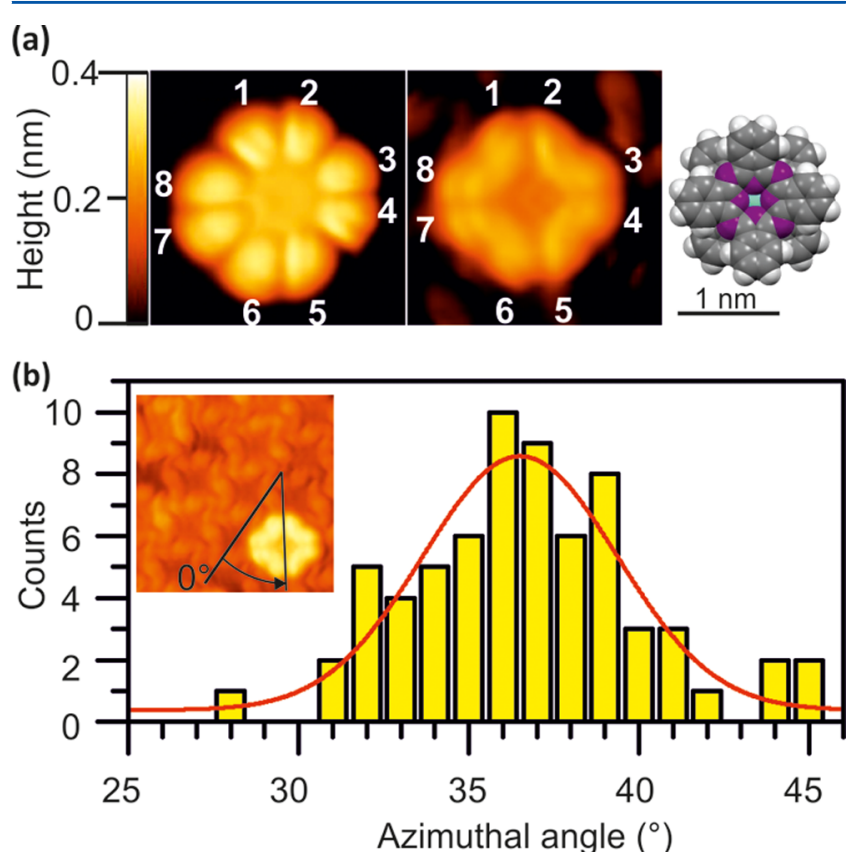

Figure 3. (a) Typical STM images of isolated single $\mathrm{TbPc}_{2}$ molecules; left: on $\mathrm{Au}(111)$ (type 4), $2.5 \times 2.5 \mathrm{~nm}^{2}, 50 \mathrm{pA},+0.9 \mathrm{~V}$; middle: adsorbed on top of a compact first-layer film of $\mathrm{TbPc}_{2}$ (type 2), $2.5 \times$ $2.5 \mathrm{~nm}^{2}, 40 \mathrm{pA},+0.9 \mathrm{~V}$; right: chemical structure model showing van der Waals atomic radii of $\mathrm{TbPc}_{2}$ as guide to the eye. (b) Histogram of azimuthal orientation angles measured on different isolated single $\mathrm{TbPc}_{2}$ molecules (type 2) lying on top of the first-layer film; a Gaussian distribution is shown in red ( $R$-square: 0.85 ); inset: definition of the azimuthal angle used for obtaining histogram (see text for details).

rule out that type 2 and type 3 molecules are triple deckers or phthalocyanines formed accidentally during evaporation: a comparison with the literature shows that triple-decker phthalocyanines exhibit significantly lower STM topographic height of about $0.55 \mathrm{~nm}$ on $\operatorname{Ag}(100)^{25}$ and $\operatorname{Au}(111){ }^{26}$ Furthermore, in contrast to the eight-lobe appearance of $\mathrm{TbPc}_{2}$ (see below), phthalocyanine molecules (5, Figure 2a) exhibit typical four-lobe appearance in topographic STM images with a height of only $0.14 \mathrm{~nm}$ on $\mathrm{Au}(111) .^{20,27}$ Based on these findings, we attribute type 2 and type 3 to intact $\mathrm{TbPc}_{2}$ molecules lying on top of the first layer, i.e., representing second-layer $\mathrm{TbPc}_{2}$ molecules.

Figure 3a juxtaposes typical topographic STM images of single isolated $\mathrm{TbPc}_{2}$ molecules adsorbed on the $\mathrm{Au}(111)$ surface (left) as well as on top of the first-layer film (right) with increased magnification; both images are displayed with the same lateral scale of $2.5 \mathrm{~nm}$ and topographical $z$-scale of 0.4 $\mathrm{nm}$. Obviously, the internal structure imaged by STM (i.e., the intramolecular contrast) appears to be very similar in both images, leading to a characteristic eight-lobe appearance. The lobes are labeled $1-8$ in the figure. As shown earlier, ${ }^{2}$ the STM topographic appearance arises predominantly from the density of states of the upper phthalocyanine ligand of $\mathrm{TbPc}_{2}$. Notice that the eight lobes are not equispaced but rather form four pairs with closer spacing (1-2, 3-4, 5-6, and 7-8). A close inspection of Figure $3 \mathrm{a}$ reveals that the lateral diameter of isolated single $\mathrm{TbPc}_{2}$ molecules, measured as full width at halfmaximum of the topographic profile, is about $10 \%$ smaller in the case of the second layer than in the first layer. As a guide to the eye for comparing the lateral sizes of molecules, Figure 3a, right, displays a chemical structure model of $\mathrm{TbPc}_{2}$ (top view) with van der Waals atomic radii and scaled 1:1 with respect to the STM images.

The second-layer $\mathrm{TbPc}_{2}$ molecules are found to lie on top of first-layer molecules with their centers aligned (Figure 2a); a similar behavior has been reported for lutetium double-decker molecules. ${ }^{28}$ A detailed topographic analysis of our STM (a)

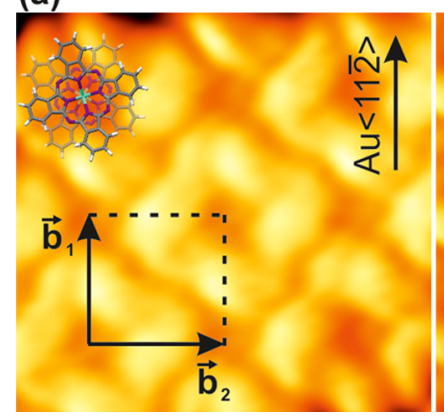

(b)

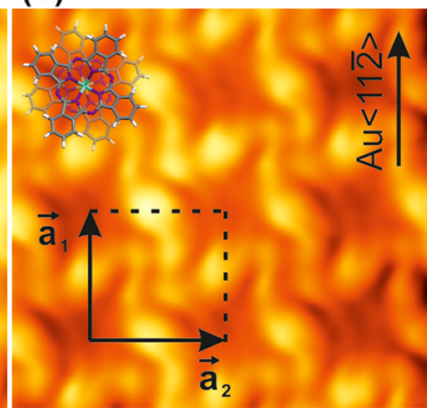

Figure 4. STM images $\left(4.5 \times 4.5 \mathrm{~nm}^{2}\right)$ of the (a) $2 \mathrm{nd} \mathrm{TbPc}_{2}$ layer $(40$ $\mathrm{pA},+0.7 \mathrm{~V})$ and (b) 1 st $\mathrm{TbPc}_{2}$ layer $(40 \mathrm{pA},+0.9 \mathrm{~V})$; the $\mathrm{Au}\langle 11 \overline{2}\rangle$ direction is indicated in both images; the 1st- and 2nd-layer molecules are ordered with the same square-like pattern with lattice vectors $\vec{a}_{1}$, $\vec{a}_{2}\left(\vec{b}_{1}, \vec{b}_{2}\right)$ indicated by arrows. Schematic models of the $\mathrm{TbPc}_{2}$ molecules are shown as a guide to the eye.

images reveals that the isolated single $\mathrm{TbPc}_{2}$ molecules of the second layer (type 2) exhibit different azimuthal orientations relative to the underlying first molecular layer. We have determined the values of the azimuthal angles of more than 65 type 2 molecules. Here, the azimuthal angle is defined as the angle between the high-symmetry axes of second- and first-layer molecules, as depicted in the inset of Figure $3 \mathrm{~b}$. A rather high variation of angles between 28 and $45^{\circ}$ is found, as illustrated by the histogram of Figure $3 \mathrm{~b}$. The reported data are fitted with a Gaussian distribution (red line in Figure $3 \mathrm{~b}$ ). The maximum of the distribution lies at $36.5^{\circ}$. In contrast, $\mathrm{TbPc}_{2}$ molecules within compact films of the second layer (type 3) are found to exhibit almost identical azimuthal orientation of about $45^{\circ}$.

A comparison between compact films of $\mathrm{TbPc}_{2}$ molecules in the second and first layer is shown in Figure $4 a$ and $b$, 
respectively. The images have been recorded at slightly different bias voltages of +0.7 and $+0.9 \mathrm{~V}$, respectively, which correspond to the energies of strong unoccupied molecular orbital states of the second- and first-layer $\mathrm{TbPc}_{2}$ molecules (discussed in detail below, see Figure 5). As clearly seen in Figure 4, the first and

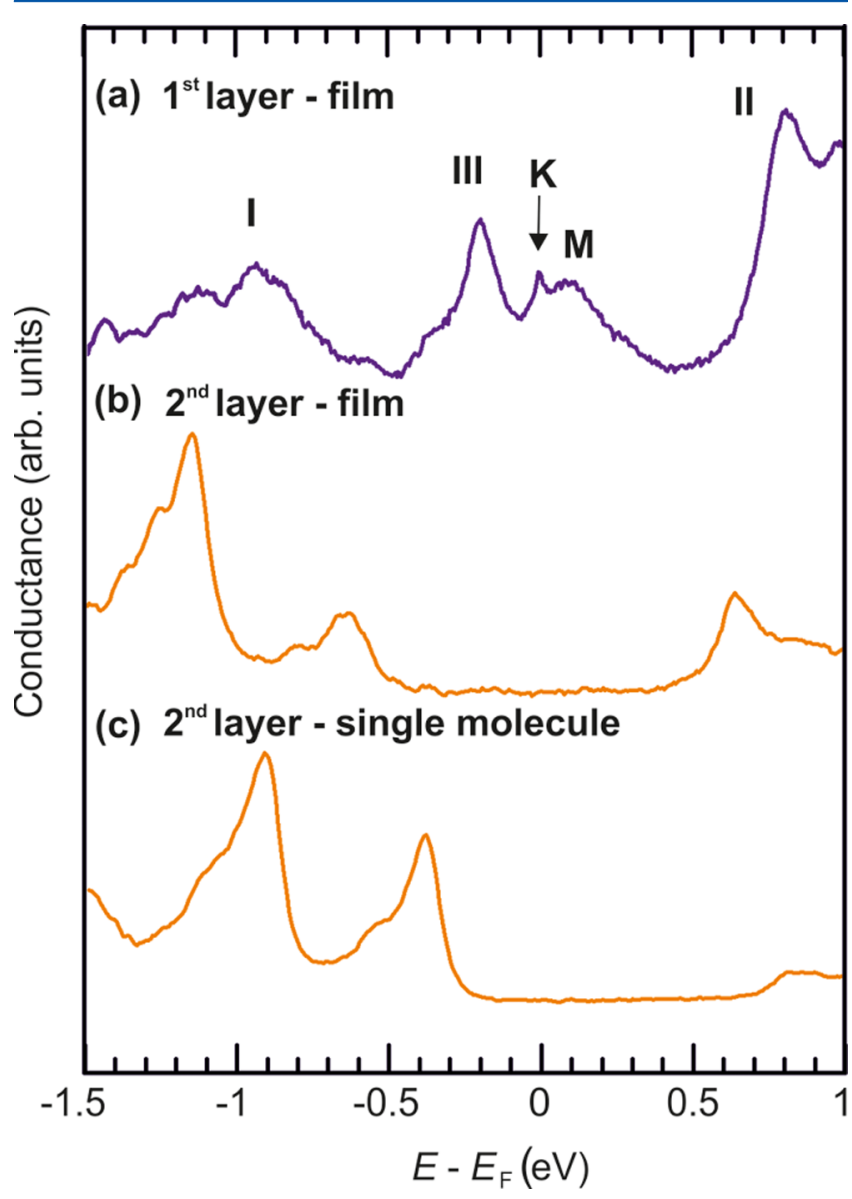

Figure 5. Experimental $d I / d V$ curves of $\mathrm{TbPc}_{2}$ in the 1st layer (A) and 2nd layer film (B) and isolated molecule (C). Prominent features are labeled I, II, III, K, and M (see text).

second layers exhibit very similar packing structures, both characterized by a square-like unit cell of approximately $1.4 \times$ $1.4 \mathrm{~nm}^{2}$. The lattice vectors are labeled $\vec{a}_{1,2}$ and $\vec{b}_{1,2}$, respectively. A schematic model of the $\mathrm{TbPc}_{2}$ molecule is superimposed in Figure 4. The bottom phthalocyanine ligand is shown in transparency for clarity.

The reduced height observed for second-layer molecules may point to a changed electronic coupling compared to the first layer. To investigate the electronic coupling, we have studied the frontier-orbital related electronic properties of first- and second-layer $\mathrm{TbPc}_{2}$ molecules by spatially resolved scanning tunneling spectroscopy. Figure 5 displays typical energy spectra of the differential tunnel conductance $(d I / d V)$ recorded on first- and second-layer molecules, respectively (with the STM tip placed over one of the eight-lobe features). The curve of Figure 5a shows a typical spectrum of $\mathrm{TbPc}_{2}$ within the firstlayer film (type 1). It exhibits pronounced peaks, consistent with the literature, at about $-0.9 \mathrm{eV}$ (I), $+0.8 \mathrm{eV}$ (II), and -0.2 $\mathrm{eV}$ (III). Peaks similar to I and II have been previously assigned to the highest occupied and lowest unoccupied molecular orbitals, respectively, ${ }^{2,11,13,29}$ and a peak similar to III has been attributed to the singly occupied molecular orbital of $\mathrm{TbPc}_{2}{ }^{13}$
The latter has been reported to exhibit a rather pronounced variation of its energy between about -0.1 and $-0.5 \mathrm{eV}$. Here, we have observed a similar large energy variation of peak III (see Supporting Information) consistent with ref 13, where the energy variation has been attributed to a varying moleculesubstrate interaction within the first-layer film due to a partial screening effect of the surrounding molecules. In Figure 5a, close to the Fermi level a sharp zero-bias peak is observed, labeled K. It originates from the Kondo resonance ${ }^{30,31}$ of an unpaired electron $(S=1 / 2)$ sitting in the $\pi$-orbital of the bottom phthalocyanine ligand, which interacts with the electron system of the $\mathrm{Au}(111)$ substrate. ${ }^{2}$ In addition, a broad peak, labeled $\mathrm{M}$, is observed close to $+0.1 \mathrm{eV}$ that was previously assigned to the electronic contribution of the metal substrate ${ }^{13,29}$ - in particular to hybrid electronic states of firstlayer $\mathrm{TbPc}_{2}$ molecules and $\mathrm{Au}(111) .^{13}$

The curve of Figure $5 \mathrm{~b}$ displays a typical $d I / d V$ spectrum of second-layer molecules in films (type 3). Intriguingly, no conductance features are observed close to the Fermi level, which is in marked contrast to the first-layer molecules (compare curves $\mathrm{a}$ and $\mathrm{b}$ of Figure 5 ). In other words, neither the Kondo resonance $(\mathrm{K})$ nor a hybrid electronic state $(\mathrm{M})$ with strong contributions of both the substrate and molecule are found in the $d I / d V$ spectrum of second-layer molecules. Similarly, the spectra of isolated single molecules of the second layer also lack both $\mathrm{K}$ and $\mathrm{M}$, as clearly shown in Figure 5c. Since $\mathrm{K}$ and $\mathrm{M}$, both, originate from the electronic interaction of $\mathrm{TbPc}_{2}$ molecules and $\mathrm{Au}(111)$ substrate, their observed absence in Figure $5 b$ indicates that such interactions are negligible for second-layer $\mathrm{TbPc}_{2}$ molecules. Notice that we have intentionally placed the STM tip over the ligand position for comparing the local spectroscopic features of first- and second-layer molecules because $\mathrm{K}$ is well-known to relate to an unpaired $\pi$-electron spin located on the phthalocyanine ligand, rather than the metallic center. ${ }^{2,13}$ While $\mathrm{K}$ is of magnetic origin (many-body spin scattering ${ }^{30,31}$ ), in contrast, $\mathrm{M}$ originates from the hybridization of substrate and first-layer molecular electronic states. ${ }^{13,29}$ As shown previously, the observed absence of $\mathrm{K}$ may indicate only a different interaction of the ligands of first- and second-layer molecules since they are known to participate deeply to the magnetic properties of $\mathrm{TbPc}_{2}$. ${ }^{2,23}$ However, the additionally observed absence of $\mathrm{M}$ indicates a decreased electronic coupling of second-layer molecules with the substrate. Our finding is in line with earlier results on multilayer iron-phthalocyanine molecules on $\operatorname{Ag}(111)$, where the vanishing of the electronic contribution from the substrate was reported to occur already in the third phthalocyanine layer. ${ }^{17}$

In addition to the lack of substrate-related peaks close to $E_{\mathrm{F}}$, the spectra of second-layer molecules are clearly distinguished by the different energies of their frontier-orbital-related peaks. The compact second layer of $\mathrm{TbPc}_{2}$ (type 3 , curve b) exhibits a pronounced conductance peak at $-1.15 \mathrm{eV}$ together with two peaks located almost symmetrically above and below $E_{\mathrm{F}}$ at $\pm 0.65 \mathrm{eV}$. The symmetric peaks apparently correspond to the highest occupied and lowest unoccupied electronic levels. Similar energies have been earlier reported for a bilayer of lutetium double-decker molecules on highly oriented pyrolytic graphite. $^{28}$ Intriguingly, the symmetry of occupied and unoccupied level energies is absent in the case of isolated single molecules (type 2) as shown in curve c. Compared to curve $b$, the whole spectrum of curve $c$ appears to be shifted by about $0.25 \mathrm{eV}$ toward the positive side. Apparently, this is a 
consequence of the lack of second-layer molecule-molecule interactions. Finally, we remark that the peak energies observed in various different second-layer $\mathrm{TbPc}_{2}$ molecules of both type 2 and type 3 have shown only variation smaller than $\pm 0.1 \mathrm{eV}$. This is in marked contrast to the more pronounced variation of more than $\pm 0.2 \mathrm{eV}$ observed for peak III of first-layer molecules (see Supporting Information). Obviously, the electronic level energies of the second-layer molecules are better defined.

\section{CONCLUSIONS}

In conclusion, we present experimental results obtained by scanning tunneling microscopy and spectroscopy on the structural and electronic properties of second-layer $\mathrm{TbPc}_{2}$ molecules, i.e., $\mathrm{TbPc}_{2}$ molecules adsorbed on top of a compact monolayer of $\mathrm{TbPc}_{2}$ on $\mathrm{Au}(111)$. Our results indicate that the first monolayer of $\mathrm{TbPc}_{2}$ decreases the electronic coupling of second-layer molecules with the $\mathrm{Au}(111)$ substrate. This is evidenced by the observed (i) absence of electronic states originating from molecule-substrate interactions close to the Fermi level and (ii) negligible variation of the energy of frontier-orbital-related states. In this respect, second-layer $\mathrm{TbPc}_{2}$ molecules exhibit frontier-orbital electronic properties similar to the gas phase.

\section{ASSOCIATED CONTENT}

\section{S Supporting Information}

The Supporting Information is available free of charge on the ACS Publications website at DOI: 10.1021/acs.jpcc.6b03676.

Experimental $d I / d V$ spectra of 1st-layer $\mathrm{TbPc}_{2}$ molecules in film on $\mathrm{Au}(111)$ (PDF)

\section{AUTHOR INFORMATION}

\section{Corresponding Author}

*E-mail: giulia.serrano@jku.at. Phone: +43 73224689643. Fax: +4373224689696.

\section{Notes}

The authors declare no competing financial interest.

\section{ACKNOWLEDGMENTS}

We kindly acknowledge financial support of the project DACH i958 by the Austrian Science Fund (FWF).

\section{REFERENCES}

(1) Ishikawa, N.; Sugita, M.; Wernsdorfer, W. Quantum Tunneling of Magnetization in Lanthanide Single-Molecule Magnets: Bis(phthalocyaninato)terbium and Bis(phthalocyaninato)dysprosium Anions. Angew. Chem., Int. Ed. 2005, 44, 2931-2935.

(2) Komeda, T.; Isshiki, H.; Liu, J.; Zhang, Y. F.; Lorente, N.; Katoh, K.; Breedlove, B. K.; Yamashita, M. Observation and Electric Current Control of a Local Spin in a Single-Molecule Magnet. Nat. Commun. 2011, 2, 217.

(3) Urdampilleta, M.; Klyatskaya, S.; Cleuziou, J. P.; Ruben, M.; Wernsdorfer, W. Supramolecular Spin Valves. Nat. Mater. 2011, 10, 502-506.

(4) Vincent, R.; Klyatskaya, S.; Ruben, M.; Wernsdorfer, W.; Balestro, F. Electronic Read-Out of a Single Nuclear Spin Using a Molecular Spin Transistor. Nature 2012, 488, 357-360.

(5) Mannini, M.; Bertani, F.; Tudisco, C.; Malavolti, L.; Poggini, L.; Misztal, K.; Menozzi, D.; Motta, A.; Otero, E.; Ohresser, P.; et al. Magnetic Behaviour of $\mathrm{TbPc}_{2}$ Single-Molecule Magnets Chemically Grafted on Silicon Surface. Nat. Commun. 2014, 5, 4582.

(6) Vitali, L.; Fabris, S.; Mosca Conte, A.; Brink, S.; Ruben, M.; Baroni, S.; Kern, K. Electronic Structure of Surface-supported
Bis(phthalocyaninato) Terbium(III) Single Molecular Magnets. Nano Lett. 2008, 8, 3364-3368.

(7) Lodi Rizzini, A.; Krull, C.; Balashov, T.; Kavich, J. J.; Mugarza, A.; Miedema, P. S.; Thakur, P. K.; Sessi, V.; Klyatskaya, S.; Ruben, M.; et al. Coupling Single Molecule Magnets to Ferromagnetic Substrates. Phys. Rev. Lett. 2011, 107, 177205.

(8) Klar, D.; Klyatskaya, S.; Candini, A.; Krumme, B.; Kummer, K.; Ohresser, P.; Corradini, V.; de Renzi, V.; Biagi, R.; Joly, L.; et al. Antiferromagnetic Coupling of $\mathrm{TbPc}_{2}$ Molecules to Ultrathin $\mathrm{Ni}$ and Co Films. Beilstein J. Nanotechnol. 2013, 4, 320-324.

(9) Klar, D.; Candini, A.; Joly, L.; Klyatskaya, S.; Krumme, B.; Ohresser, P.; Kappler, J. P.; Ruben, M.; Wende, H. Hysteretic Behaviour in a Vacuum Deposited Submonolayer of Single Ion Magnets. Dalton Trans. 2014, 43, 10686.

(10) Gonidec, M.; Biagi, R.; Corradini, V.; Moro, F.; De Renzi, V.; del Pennino, U.; Summa, D.; Muccioli, L.; Zannoni, C.; Amabilino, D. B.; et al. Surface Supramolecular Organization of a Terbium (III) Double-Decker Complex on Graphite and its Single Molecule Magnet Behavior. J. Am. Chem. Soc. 2011, 133, 6603-6612.

(11) Müllegger, S.; Tebi, S.; Das, A. K.; Schöfberger, W.; Faschinger, F.; Koch, R. Radio Frequency Scanning Tunneling Spectroscopy for Single-Molecule Spin Resonance. Phys. Rev. Lett. 2014, 113, 133001.

(12) Thiele, S.; Balestro, F.; Ballou, R.; Klyatskaya, S.; Ruben, M.; Wernsdorfer, W. Electrically Driven Nuclear Spin Resonance in SingleMolecule Magnets. Science 2014, 344, 1135-1138.

(13) Komeda, T.; Isshiki, H.; Liu, J.; Katoh, K.; Yamashita, M. Variation of Kondo Temperature Induced by Molecule-Substrate Decoupling in Film Formation of Bis(phthalocyaninato)terbium(III) Molecules on $\mathrm{Au}(111)$. ACS Nano 2014, 8, 4866-4875.

(14) Qiu, X.; Nazin, G.; Ho, W. Vibrationally Resolved Fluorescence Excited with Submolecular Precision. Science 2003, 299, 542-546.

(15) Ćavar, E.; Blüm, M.-C.; Pivetta, M.; Patthey, F.; Chergui, M.; Schneider, W.-D. Fluorescence and Phosphorescence From Individual $\mathrm{C}_{60}$ Molecules Excited by Local Electron Tunneling. Phys. Rev. Lett. 2005, 95, 196102.

(16) Guo, X.; Dong, Z.; Trifonov, A.; Miki, K.; Mashiko, S.; Okamoto, T. Molecular Fluorescence from ZnTBP Porphyrin Molecular Layers on $\mathrm{Cu}(100)$ Induced by Tunnelling Currents. Nanotechnology 2004, 15, S402.

(17) Gopakumar, T.; Brumme, T.; Kroger, J.; Toher, C.; Cuniberti, G.; Berndt, R. Coverage-Driven Electronic Decoupling of $\mathrm{Fe}$ Phthalocyanine From a $\mathrm{Ag}(111)$ Substrate. J. Phys. Chem. C 2011, $115,12173-12179$.

(18) Franke, K.; Schulze, G.; Henningsen, N.; Fernández-Torrente, I.; Pascual, J.; Zarwell, S.; Rück-Braun, K.; Cobian, M.; Lorente, N. Reducing the Molecule-Substrate Coupling in $\mathrm{C}_{60}$-Based Nanostructures by Molecular Interactions. Phys. Rev. Lett. 2008, 100, 036807.

(19) Zhang, Y. F.; Isshiki, H.; Katoh, K.; Yoshida, Y.; Yamashita, M.; Miyasaka, H.; Breedlove, B. K.; Kajiwara, T.; Takaishi, S.; Komeda, T. Low-Temperature Scanning Tunneling Microscopy Investigation of $\mathrm{Bis}$ (phthalocyaninato)yttrium Growth on $\mathrm{Au}(111)$ : From Individual Molecules to Two-Dimensional Domains. J. Phys. Chem. C 2009, 113, 9826-9830.

(20) Katoh, K.; Yoshida, Y.; Yamashita, M.; Miyasaka, H.; Breedlove, B. K.; Kajiwara, T.; Takaishi, S.; Ishikawa, N.; Isshiki, H.; Zhang, Y. F.; et al. Direct Observation of Lanthanide(III)-Phthalocyanine Molecules on $\mathrm{Au}(111)$ by Using Scanning Tunneling Microscopy and Scanning Tunneling Spectroscopy and Thin-Film Field-Effect Transistor Properties of $\mathrm{Tb}(\mathrm{III})$ - and $\mathrm{Dy}(\mathrm{III})-$ Phthalocyanine Molecules. J. Am. Chem. Soc. 2009, 131, 9967-9976.

(21) Komeda, T.; Katoh, K.; Yamashita, M. Double-Decker Phthalocyanine Complex: Scanning Tunneling Microscopy Study of Film Formation and Spin Properties. Prog. Surf. Sci. 2014, 89, 127160

(22) Schwöbel, J.; Fu, Y.; Brede, J.; Dilullo, A.; Hoffmann, G.; Klyatskaya, S.; Ruben, M.; Wiesendanger, R. Real-Space Observation of Spin-Split Molecular Orbitals of Adsorbed Single-Molecule Magnets. Nat. Commun. 2012, 3, 953. 
(23) Fu, Y.-S.; Schwöbel, J.; Hla, S.-W.; Dilullo, A.; Hoffmann, G.; Klyatskaya, S.; Ruben, M.; Wiesendanger, R. Reversible Chiral Switching of Bis(phthalocyaninato) Terbium(III) on a Metal Surface. Nano Lett. 2012, 12, 3931-3935.

(24) Deng, Z.; Rauschenbach, S.; Stepanow, S.; Klyatskaya, S.; Ruben, M.; Kern, K. Self-Assembly of Bis(phthalocyaninato)terbium on Metal Surfaces. Phys. Scr. 2015, 90, 098003.

(25) Lodi Rizzini, A.; Krull, C.; Mugarza, A.; Balashov, T.; Nistor, C.; Piquerel, R.; Klyatskaya, S.; Ruben, M.; Sheverdyaeva, P. M.; Moras, P.; et al. Coupling of Single, Double, and Triple-Decker MetalPhthalocyanine Complexes to Ferromagnetic and Antiferromagnetic Substrates. Surf. Sci. 2014, 630, 361-374.

(26) Isshiki, H.; Liu, J.; Katoh, K.; Yamashita, M.; Miyasaka, H.; Breedlove, B. K.; Takaishi, S.; Komeda, T. Scanning Tunneling Microscopy Investigation of Tris(phthalocyaninato)yttrium TripleDecker Molecules Deposited on Au(111). J. Phys. Chem. C 2010, 114, 12202-12206.

(27) Nilson, K.; Åhlund, J.; Brena, B.; Göthelid, E.; Schiessling, J.; Mårtensson, N.; Puglia, C. Scanning Tunneling Microscopy Study of Metal-Free Phthalocyanine Monolayer Structures on Graphite. J. Chem. Phys. 2007, 127, 114702.

(28) Smykalla, L.; Shukrynau, P.; Hietschold, M. Investigation of Ultrathin Layers of Bis(phthalocyaninato)lutetium (III) on Graphite. J. Phys. Chem. C 2012, 116, 8008-8013.

(29) Müllegger, S.; Rauls, E.; Gerstmann, U.; Tebi, S.; Serrano, G.; Wiespointner-Baumgarthuber, S.; Schmidt, W. G.; Koch, R. Mechanism for Nuclear and Electron Spin Excitation by Radio Frequency Current. Phys. Rev. B: Condens. Matter Mater. Phys. 2015, 92, 220418.

(30) Madhavan, V.; Chen, W.; Jamneala, T.; Crommie, M. F.; Wingreen, N. S. Tunneling into a Single Magnetic Atom: Spectroscopic Evidence of the Kondo Resonance. Science 1998, 280, 567.

(31) Li, J.; Schneider, W. D.; Berndt, R.; Delley, B. Kondo Scattering Observed at a Single Magnetic Impurity. Phys. Rev. Lett. 1998, 80, 2893-2896. 